\title{
Entzündungshemmer verhindert Herzinfarkte
}

\author{
Erstmals ist es gelungen, mit einem antientzündlichen Therapieansatz die Prognose von \\ Herzpatienten zu verbessern. In der CANTOS-Studie reduzierte der Antikörper Canakinumab \\ das relative Risiko für kardiovaskuläre Komplikationen um $15 \%$.
}

_ In der von Novartis unterstützten „Canakinumab Antiinflammatory Thrombosis Outcome Study“ (CANTOS) war für eine mediane Laufzeit von 3,7 Jahren den Patienten alle drei Monate der gegen Interleukin-1 $\beta$ gerichtete monoklonale Antikörper Canakinumab $\left(\right.$ Ilaris $\left.^{\circledast}\right)$ s.c. appliziert worden.

Die 10.061 Studienteilnehmer hatten sich durch eine Herzinfarkt-Anamnese und hs-CRP-Werte über $2 \mathrm{mg} / \mathrm{l}$ qualifiziert. Die Patienten wiesen zahlreiche kardiovaskuläre Risikofaktoren auf. Über 90\% nahmen Statine ein, viele waren mehrfach revaskularisiert worden. Behandelt wurde in vier Gruppen (Canakinumab 50 mg, 150 mg, 300 mg, Placebo).

\section{Dosisabhängiger hs-CRP-Rückgang}

Die Antikörper-Therapie reduzierte den CRP-Wert dosisabhängig: um 26\% unter $50 \mathrm{mg}$, um $37 \%$ unter $150 \mathrm{mg}$ und um $41 \%$ unter $300 \mathrm{mg}$. Die 150-mg-Dosis führte im Studienzeitraum zu einer sta- tistisch signifikanten relativen Risikosenkung für klinische Ereignisse (14\% vs. $16 \%$ unter Placebo). Je stärker das CRP abfiel, desto größer der klinische Nutzen.

\section{Moderater klinischer Effekt, aber das Prinzip ist bewiesen}

Der Effekt war „moderat“, so Prof. R. Harrington von der Stanford University. Allerdings beweise die Studie, dass die Inflammation bei Atherosklerose nicht nur pathogenetisch eine Rolle spielt, sondern auch therapeutisch ein Ziel sein kann.

Prof. Wolfgang König vom Herzzentrum München spricht von der „Dualtarget-Hypothese“: Trotz optimal kontrollierter LDL-Cholesterin-Werte verbleibe ein Restrisiko für Herzinfarkte, für das möglichkerweise eine subklinische Inflammation verantwortlich ist. Die Canakinumab-Studie beweist nun das wissenschaftliche Prinzip. Der moderate klinische Nutzen der bis dato sehr teuren Therapie rechtfertigt noch nicht den brei- ten Einsatz bei Infarktpatienten; zuvor müssen Sicherheit und Wirksamkeit besser untersucht werden, so Harrington.

\section{Nebenwirkungen: Infektionsrisiko erhöht, Krebssterblichkeit erniedrigt} Bezüglich der Sicherheit fiel auf, dass die Therapie das Risiko für tödliche Infektionen erhöhte $(0,18 \%$ unter Placebo, 0,28\% unter $150 \mathrm{mg}$ Canakinumab). Andererseits senkte sie signifikant das Risiko für tödliche Krebserkrankungen (0,64\% unter Placebo; 0,5\% unter $150 \mathrm{mg}$ Canakinumab). Die 300-mg-Dosis halbierte gar die Krebssterblichkeit. Verhindert wurden vor allem Bronchialkarzinome. Unter dem Strich blieb ein „neutraler Effekt“ auf die Sterblichkeit.

Dr. Dirk Einecke

- 1. ESC-Kongress, Barcelona 26.-30. August 2017 2. Ridker PM, et al. Antiinflammatory Therapy with Canakinumab for Atherosclerotic Disease. N Engl J Med 2017; https://doi: 10.1056/NEJMoa1707914

3. Harrington RA; Targeting Inflammation in Coronary Artery Disease. N Eng/J Med 2017; https://doi: 10.1056/NEJMoa1709904

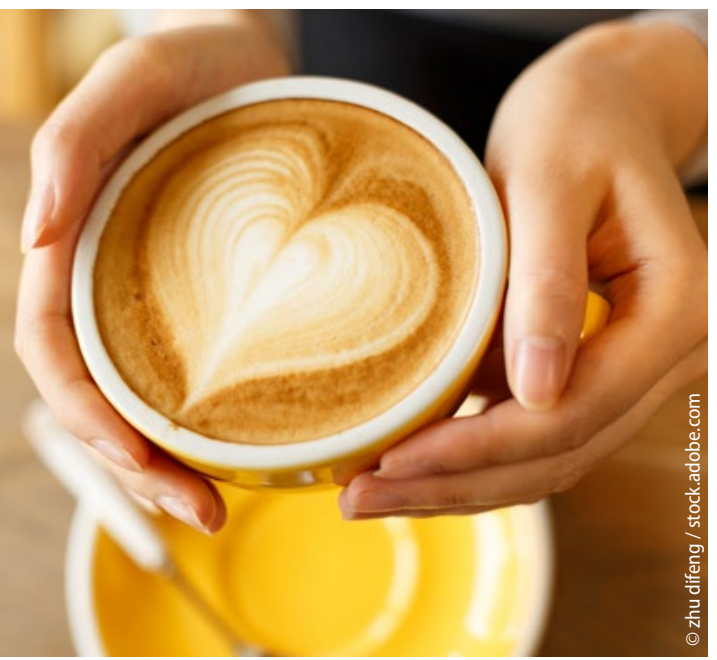

\section{Hoher Kaffeekonsum fördert die Langlebigkeit}

Gute Nachricht für alle Kaffee-Liebhaber: Wer viel von diesem anregenden Gebräu trinkt, lebt länger, lautet das erfreuliche Ergebnis einer großen spanischen Studie.

Die Daten stammen aus dem "Seguimiento Universidad de Navarra“-Projekt, bei dem knapp 20.000 zu Beginn im Schnitt 38 Jahre alte Menschen über durchschnittlich zehn Jahre prospektiv beobachtet wurden. 337 Teilnehmer starben während des Follow-up. Mit dem Kaffeegenuss nahm die Langlebigkeit zu: Wer vier oder mehr Tassen Kaffee pro Tag trank, hatte ein relativ um 64\% geringeres Sterberisiko als Personen, die nie oder nur selten Kaffee konsumierten.

Mit jeder Tasse mehr pro Tag, so die Autoren, nahm die Sterblichkeit um 22\% ab. Die protektive Beziehung war bei Personen im Alter über 45 Jahren besonders ausgeprägt (30\%ige Mortalitätsreduktion pro täglich getrunkener Tasse Kaffee). Die Autoren gehen so weit zu fordern, dass „vier Tassen Kaffee täglich“ eine Komponente der Empfehlungen für eine gesunde Ernährung sein sollten.

- DE 